\title{
ADMISSIBLE KERNELS FOR STARSHAPED SETS
}

\author{
MARILYN BREEN ${ }^{1}$
}

\begin{abstract}
Steven Lay has posed the following interesting question: If $D$ is a convex subset of $\mathbf{R}^{d}$, then is there a starshaped set $S \neq D$ in $\mathbf{R}^{d}$ whose kernel is $D$ ? Thus the problem is that of characterizing those convex sets which are admissible as the kernel of some nonconvex starshaped set in $\mathbf{R}^{d}$. Here Lay's problem is investigated for closed sets, and the following results are obtained: If $D$ is a nonempty closed convex subset of $\mathbf{R}^{2}$, then $D$ is the kernel of some planar set $S \neq D$ if and only if $D$ contains no line. If $D$ is a compact convex set in $R^{d}$, then there is a compact set $S \neq D$ in $\mathbf{R}^{d}$ whose kernel is $D$.
\end{abstract}

1. Introduction. Let $S$ be a subset of $\mathbf{R}^{d}$. Set $S$ is said to be starshaped if and only if there is some point $p$ in $S$ such that $[p, x] \subseteq S$ for every $x$ in $S$. The set of all such points $p$ is called the (convex) kernel of $S$, denoted ker $S$. While many interesting results have been obtained for starshaped sets and their kernels [2], a new problem has been posed recently by Steven Lay [1]. If $D$ is a convex subset of $\mathbf{R}^{d}$, then is there a starshaped set $S \neq D$ in $\mathbf{R}^{d}$ whose kernel is $D$ ? That is, can we characterize those convex sets which are admissible as the kernel of some nonconvex starshaped set in $\mathbf{R}^{d}$ ? The question may be answered quickly in various special cases. In particular, when $D$ is not a $(d-1)$-flat and $D$ has dimension less than $d$, it is not hard to construct an appropriate set $S$. Similarly, when $D$ is a polytope, such an $S$ exists. However, the problem for arbitrary $D$ is both intriguing and challenging. Here we begin by considering a closed convex set $D$ in the plane, and constructions for $S$ are given when $D$ contains no line. If $D$ is closed and contains a line, then it is proved that no such set $S$ exists. Finally, extensions from $\mathbf{R}^{2}$ to $\mathbf{R}^{d}$ are obtained with certain modifications.

The following definitions and terminology will be used throughout the paper. Let $S$ be a subset of $\mathbf{R}^{d}$. A point $x$ in $S$ is said to be a point of local convexity of $S$ if and only if there is some neighborhood $N$ of $x$ such that $N \cap S$ is convex. In case $S$ fails to be locally convex at point $q$ in $S$, then $q$ is called a point of local nonconvexity (lnc point) of $S$. For $S$ a 2-dimensional convex set, line $L$ is said to support $S$ if and only if $L$ contains no interior point of $S$ and the distance from $L$ to $S$ is zero. (Notice that such a line need not meet $S$.) The terms conv $S$, aff $S$, cl $S$, bdry $S$, and int $S$ will denote the convex hull, affine hull, closure, boundary, and interior of set $S$, respectively. When $S$ is convex, $\operatorname{dim} S$ will be the dimension of set

\footnotetext{
Received by the editors August 11, 1980 and, in revised form, October 7, 1980.

1980 Mathematics Subject Classification. Primary 52A10, 52A30, 52A20.

Key words and phrases. Starshaped sets, convex kernel.

1 This research was sponsored in part by an Arts and Sciences summer fellowship from the University of Oklahoma.
} 
$S$. For distinct points $x$ and $y$, dist $(x, y)$ will represent the distance from $x$ to $y$, $L(x, y)$ will represent the line determined by $x$ and $y$, and $R(x, y)$ will be the ray emanating from $x$ through $y$.

2. The results in $\mathbf{R}^{2}$. We begin with the following theorem for compact convex sets in $\mathbf{R}^{2}$.

THEOREM 1. Let $D$ be any nonempty compact convex set in $\mathbf{R}^{2}$. Then there is a compact set $S \neq D$ in $\mathbf{R}^{2}$ with $\operatorname{ker} S=D$.

Proof. We begin by disposing of the cases for $\operatorname{dim} D<2$. If $D$ is a singleton set, let $S$ be the union of two closed disks whose intersection is exactly $D$. If $D$ is a segment $[x, y]$, choose $x_{0}, y_{0}$ on $L(x, y)$ with $x_{0}<x<y<y_{0}$, choose $z \notin L(x, y)$, and let $S=\operatorname{conv}\{x, y, z\} \cup\left[x_{0}, y_{0}\right]$.

Throughout the remainder of the argument, we assume that $D$ is a full 2-dimensional set. For convenience of notation, we suppose that $D$ lies in the unit disk $\{z$ : $|z|=1\}$, with the origin $\theta$ interior to $D$. Moreover, assume that $D$ is oriented so that it has a horizontal line of support which intersects the set on the positive $y$ axis. Let $T_{L}$ and $T_{R}$ denote the vertical lines which support $D ; T_{L}$ in the left open halfplane, $T_{R}$ in the right open halfplane. For each point $x \notin D$, there are two distinct segments $\left(x, y_{1}\right)$ and $\left(x, y_{2}\right)$ disjoint from $D$ whose lines support $D$ at $y_{1}$ and $y_{2}$, respectively. We call $\left[x, y_{1}\right]$ the left supporting segment from $x$ to $D$ and call $\left[x, y_{2}\right]$ the right supporting segment from $x$ to $D$ provided that the order along bdry conv $\left(y_{1}, x, y_{2}\right)$ in a clockwise direction from $y_{1}$ gives $y_{1}, x, y_{2}$. A parallel definition holds for left and right supporting lines $L\left(x, y_{1}\right)$ and $L\left(x, y_{2}\right)$.

We will construct set $S$ inductively, using an increasing sequence of closed sets. Choose a point $p_{1}$ not in $D$ and in the open convex region bounded by $T_{L}$ and $T_{R}$. For the sake of simplicity, let $p_{1}$ be a point on the $y$ axis for $y>2$. Define $S_{1}=\operatorname{conv}\left(p_{1} \cup D\right)$. Let $w_{1}$ be the midpoint of the left supporting segment $\left[p_{1}, s_{1}\right]$ from $p_{1}$ to $D$. Let $T_{1}$ be the right supporting line from $w_{1}$ to $D$ at point $s_{2}$. Choose the point $u_{1}$ of $T_{1}$ such that $w_{1}$ is between $u_{1}$ and $s_{2}$ and $\operatorname{dist}\left(w_{1}, u_{1}\right)$ is half the distance from $w_{1}$ to $T_{L}$. Then $\left(w_{1}, u_{1}\right) \cap S_{1}=\varnothing$. Relabel peak $p_{1}$ by $p_{21}$, peak $u_{1}$ by $p_{22} \equiv b_{2}$, and define $S_{2}=\cup\left\{\operatorname{conv}\left(p_{2 i} \cup D\right): 1<i<2\right\}$. Label the lnc point $w_{1}$ of $S_{2}$ by $s_{21}$, and let $s_{22}$ be the point of $D$ such that $\left[p_{22}, s_{22}\right]$ is the left supporting segment from $p_{22}$ to $D$.

Inductively, for $k>2$ assume that consecutive peaks $p_{k 1}, p_{k 2}, \ldots, p_{k j} \equiv b_{k}$ are defined, $j=2^{k-1}$, that $S_{k}=\cup\left\{\operatorname{conv}\left(p_{k i} \cup D\right): 1<i<j\right\}$, and that $s_{k 1}, s_{k 2}, \ldots, s_{k j}$ are defined. We obtain $S_{k+1}$ as follows. For each $i, 1<i<j$, let $w_{k i}$ be the midpoint of segment $\left[p_{k i}, s_{k i}\right]$. Let $T_{k i}$ be the right supporting line from $w_{k i}$ to $D$. For $i \neq j$, choose point $u_{k i}$ of $T_{k i}$ whose distance to $w_{k i}$ is half the distance from $w_{k i}$ to segment $\left[p_{k i}, p_{k, i+1}\right]$ along $T_{k i}$ and $\left(w_{k i}, u_{k i}\right) \cap S_{k}=\varnothing$. For $i=j$, choose point $u_{k j}$ of $T_{k j}$ such that $\operatorname{dist}\left(w_{k j}, u_{k j}\right)=\frac{1}{2} \operatorname{dist}\left(w_{k j}, T_{L}\right)$. (Recall that $T_{L}$ is the left vertical line of support to D.) Relabel peaks $p_{k 1}, u_{k 1}, p_{k 2}, u_{k 2}, \ldots, p_{k j}, u_{k j}$ by $p_{k+1,1}, p_{k+1,2}, \ldots, p_{k+1,2 j} \equiv b_{k+1}$, respectively, and refer to $p_{k+1, i}$ and $p_{k+1, i+1}$ as consecutive peaks, $1 \leqslant i \leqslant 2 j-1$. Define $S_{k+1}=\bigcup\left\{\operatorname{conv}\left(p_{k+1, i} \cup D\right): 1<i<\right.$ $2 j\}$. For future reference, notice that for each peak $p_{k+1, i}$ of $S_{k+1}$ and for $M$ a 
supporting line from $p_{k+1, i}$ to $D$, sets $\left\{p_{k+1,1}, \ldots, p_{k+1, i-1}\right\}$ and $\left\{p_{k+1, i+1}, \ldots, p_{k+1,2 j}\right\}$ lie in opposite open halfplanes determined by $M$. Thus no point $p_{k+1, i}$ is captured by a remaining convex hull conv $\left(p_{k+1, m} \cup D\right), m \neq i$. Note also that the slopes of the right supporting lines to $D$ from consecutive peaks of $S_{k+1}$ form an increasing sequence, as do the slopes of the left supporting lines. Finally, observe that $\left(p_{k+1, i}, p_{k+1, i+1}\right) \cap S_{k+1}=\varnothing$ for $1<i<2 j-1$.

Continuing our inductive construction, label the lnc points of $S_{k+1}$ by $s_{k+1,1}, s_{k+1,2}, \ldots, s_{k+1,2 j-1}$, where $s_{k+1, i}$ is the lnc point of $\operatorname{conv}\left(p_{k+1, i} \cup D\right) \cup$ $\operatorname{conv}\left(p_{k+1, i+1} \cup D\right), 1 \leqslant i<2 j-1$. And let $s_{k+1,2 j}$ be the point of $D$ such that $\left[p_{k+1,2 j}, s_{k+1,2 j}\right]$ is the left supporting segment from $p_{k+1,2 j} \equiv b_{k+1}$ to $D$. By our inductive construction, $S_{n}$ is defined for every natural number $n$. We define set $S_{L}=\cup_{1<n} S_{n}$.

Now begin again with set $S_{1} \equiv S_{1}^{\prime}$ and apply the construction above to the right halfplane, using right supporting segments (instead of left), left supporting lines (instead of right), and line $T_{R}$ (instead of $T_{L}$ ). We thereby obtain an increasing sequence of sets $\left\{S_{n}^{\prime}\right\}$, and define set $S_{R}=\cup_{1<n} S_{n}^{\prime}$. Finally, define $S=$ $\operatorname{cl}\left(S_{L} \cup S_{R}\right)$. Observe that $S_{R}$ contributes no points to $S_{L}$ in the left halfplane, $S_{L}$ contributes none to $S_{R}$ in the right.

Clearly $S$ is a compact set and $S \neq D$. We assert that ker $S=D$. It is easy to show that $D \subseteq \operatorname{ker} S$, so we will establish only the reverse inclusion. As a preliminary step, we show that for $p$ one of the peaks defined above, say $p=p_{k i}, p$ is an lnc point of $S$. Without loss of generality, assume that $p \in S_{L}$. Let $N$ be any circular neighborhood of $p$. By our construction, $N$ contains another peak, say $q=p_{l m}$, where $l>k$ and where $q$ and $\theta$ are in opposite open halfplanes determined by line $L\left(p_{k i}, s_{k i}\right)$. Moreover, $q$ may be selected so that $p$ and $q$ are consecutive peaks for $S_{l}$. Then by a previous comment, $(p, q) \cap S_{l}=\varnothing$. Select $r$ so that $p, r, q$ are consecutive peaks for $S_{l+1}$. For $n>l+1$, every peak $t \neq p, r, q$ of $S_{n}$ which lies in the convex region determined by rays $R(\theta, p)$ and $R(\theta, q)$ must lie in the open halfplane determined by $L(p, r)$ and containing $\theta$ and in the open halfplane determined by $L(r, q)$ and containing $\theta$. Thus $\operatorname{conv}(t \cup D)$ is disjoint from $\operatorname{conv}(p, r, q)$, and such a peak $t$ cannot contribute any point to $\operatorname{conv}(p, r, q)$ in $S_{n}$ or in $S$. Using previous comments, it is easy to see that the remaining peaks of $S_{n}$ cannot contribute any point to $\operatorname{conv}(p, r, q)$ either, and we conclude that $(p, q) \cap S=\varnothing$. Hence $p$ is indeed an lnc point for $S$, and our preliminary result is established.

Now we are able to show that $\operatorname{ker} S \subseteq D$. Let $x \in S \sim D$ to prove that $x \notin \operatorname{ker} S$. Without loss of generality, assume that $x$ is in the closed left halfplane. Recall that, for each $k \geqslant 2, b_{k}$ denotes the last peak of $S_{k}$ in our ordering, and clearly the sequence $\left\{b_{k}\right\}$ converges to a point of $D \cap T_{L}$. By our construction, it is not hard to see that $T_{L} \cap S=T_{L} \cap D$, so $x \notin T_{L}$. Therefore, there must be some peak $p$ in the open left halfplane such that the right supporting segment from $p$ to $D$ has slope greater than the slope of the right supporting segment $E$ from $x$ to $D$. That is, $x$ and $\theta$ lie in opposite open halfplanes $E_{1}$ and $E_{2}$, respectively, determined by the line of $E$. Again by our construction, there is a sequence of 
distinct peaks in $E_{1}$ converging to a point of $E$, and it is not hard to see that this sequence is eventually in int $\operatorname{conv}(p \cup x \cup D)$. Hence for $n$ sufficiently large, int $\operatorname{conv}(p \cup x \cup D)$ necessarily contains peaks of $S_{n}$, and since such peaks are lnc points of $S$, int $\operatorname{conv}(p \cup x \cup D)$ must contain points in $\sim S$. Therefore, $x \cup D \nsubseteq \operatorname{ker} S$, and since $D \subseteq \operatorname{ker} S$, this implies that $x \notin \operatorname{ker} S$. We conclude that ker $S \subseteq D$ and the sets are equal, completing the proof of Theorem 1 .

In case $D$ contains a ray but no line, the argument above may be adapted to obtain the following result.

THEOREM 2. Let $D$ be any closed unbounded convex set in $\mathbf{R}^{2}$ which contains no line. Then there is a closed set $S \neq D$ in $\mathbf{R}^{2}$ with $\operatorname{ker} S=D$.

Proof. If $D$ has dimension less than 2, a variation of our opening argument in Theorem 1 yields the result. Hence assume that $D$ is fully 2-dimensional. For convenience, orient $D$ so that int $D$ contains the negative $y$ axis, $D$ does not contain either the positive or the negative $x$ axis, and $D$ has a horizontal line of support at the origin. For point $x \notin D$, there are two distinct supporting lines from $x$ to $D$ (which may or may not meet $D$ ). The definitions of left and right supporting lines from $x$ to $D$ (in Theorem 1) may be adapted in an obvious manner to this case. When such a supporting line meets $D$, we may talk about the corresponding left or right supporting segment from $x$ to $D$.

We proceed as follows to define set $S$. In case $D$ is a cone, $D$ may be represented by $\cup\{R(v, c): c \in C\}$ where $C$ is some compact convex set not containing point $v$. Then it is easy to construct $S$ by creating an lnc point appropriately at $v$. For the remainder of the argument, we will assume that $D$ is not a cone. Select points $z_{L}$ and $z_{R}$ in bdry $D, z_{L}$ in the left open halfplane, $z_{R}$ in the right. Also, since $D$ is not a cone, we may choose these points so that at least one of the segments $\left[z_{L}, \theta\right]$ and $\left[z_{R}, \theta\right]$ is not in bdry $D$. Let $T_{L}$ and $T_{R}$ denote lines which support $D$ at $z_{L}$ and $z_{R}$, respectively, and let $A$ denote the open convex region determined by $T_{L}$ and $T_{R}$ and containing int $D$. Either $A$ will be the interior of a cone whose vertex lies in the upper halfplane or $A$ will be an open parallel strip.

Define $B$ to be the bounded component of (bdry $D) \sim\left(T_{L} \cup T_{R}\right)$. By our choice of $z_{L}$ and $z_{R}$, it is clear that $B$ is not empty and $\mathrm{cl} B$ contains the origin. Furthermore, if $F$ denotes the component of $A \sim D$ whose boundary contains $B$, then for $x$ in $F$, each supporting segment from $x$ to $D$ necessarily meets cl $B$. Select point $p_{1}$ in $F$ and define $S_{1}=\operatorname{conv}\left(p_{1} \cup D\right)$. Using $p_{1}, T_{L}$, and $T_{R}$ defined above, employ the inductive construction in Theorem 1. At each stage of the construction, every peak $p_{k i}$ will lie in the region $F$. With minor modifications in terminology, the previous argument may be duplicated to define the closed set $S$ and to verify that $\operatorname{ker} S=D$. This completes the proof of Theorem 2 .

If the closed set $D$ contains a line, then no appropriate set $S$ exists, as our final theorem reveals.

TheOREM 3. Let $D$ be any convex set in $\mathbf{R}^{2}$ which contains a line $L$. Then $D$ is the kernel of some planar set $S \neq D$ if and only if there is a line $M$ in bdry $D$ with $M \cap D=\varnothing$. 
Proof. We dispose of the easy case first. If bdry $D$ contains such a line $M$, select distinct points $p$ and $q$ in $M$. Defining $S=D \cup\{p, q\}$, standard arguments reveal that $D=\operatorname{ker} S$.

To establish the converse statement, assume that no such line $M$ exists. If $D=\mathbf{R}^{2}$, the result is immediate, so suppose that this is not the case. Then clearly bdry $D=H \cup J$ where $H$ and $J$ are lines parallel to $L$ (and not necessarily distinct). Furthermore, by hypothesis, $H \cap D \neq \varnothing$ and $J \cap D \neq \varnothing$. Let $S$ denote any planar set distinct from $D$ with $D \subseteq \operatorname{ker} S$. (Clearly $\mathbf{R}^{2}$ itself is such a set.) To finish the proof of Theorem 3, it suffices to show that ker $S \nsubseteq D$.

In case $S \subseteq \operatorname{cl} D$, then since $H \cap D \neq \varnothing$ and $J \cap D \neq \varnothing$, it is easy to show that $S$ is convex. Thus $\operatorname{ker} S=S \nsubseteq D$, and the theorem is proved. Therefore, throughout the remainder of the argument we may restrict our attention to the case in which $S \nsubseteq \operatorname{cl} D$.

The following notation will be useful. For each point $z$ in $S \sim L$, let $M_{z}$ denote the line through $z$ and parallel to $L$, with $P_{z}$ the open parallal strip bounded by $M_{Z}$ and $L$. Notice that since $L \subseteq D \subseteq \operatorname{ker} S, P_{z} \subseteq S$.

To show that $\operatorname{ker} S \nsubseteq D$, select a point $x$ in $S \sim \operatorname{cl} D \neq \varnothing$. There is a neighborhood $N$ of $x$ disjoint from cl $D$, and we may choose some point $x^{\prime}$ in $N \cap P_{x} \subseteq S$ $\sim \operatorname{cl} D$. We assert that $x^{\prime} \in \operatorname{ker} S$ : Choose $y \in S$ to show that $\left[x^{\prime}, y\right] \subseteq S$. If $y \in L \subseteq \operatorname{ker} S$, then it is immediate that $\left[x^{\prime}, y\right] \subseteq S$, so we assume that $y \in S \sim$ $L$. Then $P_{x} \cup P_{y} \cup L$ is a (not necessarily open) parallel strip in $S$ with $x^{\prime}$ in its interior and $y$ in its closure. Thus $\left[x^{\prime}, y\right) \subseteq P_{x} \cup P_{y} \cup L \subseteq S$ and $\left[x^{\prime}, y\right] \subseteq S$. We conclude that $x^{\prime} \in \operatorname{ker} S \sim \operatorname{cl} D \subseteq \operatorname{ker} S \sim D$ and $\operatorname{ker} S \nsubseteq D$. The proof of Theorem 3 is established.

We conclude the planar case with two corollaries. The first may be proved using an argument from Theorem 3 above.

Corollary 1. Let $D$ be any convex set in $\mathbf{R}^{2}$ which contains a line. Then $D$ is not the kernel of any planar set which property contains cl $D$.

The second corollary, our characterization theorem, is an immediate consequence of Theorems 1, 2, and 3.

Corollary 2. Let $D$ be a nonempty closed convex subset of $\mathbf{R}^{2}$. Then $D$ is the kernel of some planar set $S \neq D$ if and only if $D$ contains no line.

Notice that Corollary 2 above fails without the requirement that $D$ be closed, as Theorem 3 reveals.

3. The results in $\mathbf{R}^{d}$. Theorem 1 may be modified to obtain the following extension from $\mathbf{R}^{2}$ to $\mathbf{R}^{d}$.

THEOREM 4. Let $D$ be a nonempty compact set in $\mathbf{R}^{d}, d>2$. Then there is a compact set $S \neq D$ in $\mathbf{R}^{d}$ with $\operatorname{ker} S=D$.

Proof. The inductive construction for $S$ is outlined below. We restrict our attention to $\mathbf{R}^{d}$ for $d \geqslant 3$, and assume that $D$ is fully $d$-dimensional. Consider the family $\mathscr{F}$ of closed halfspaces of the form $[\alpha: a] \equiv\left\{z: z \in \mathbf{R}^{d}\right.$ and $\left.\alpha \cdot z>a\right\}$, 
where $\alpha$ is any $d$-dimensional vector having rational coordinates and where $a$ is any rational scalar. It is known that $\mathscr{F}$ is an intersectional basis for the set of compact convex subsets of $\mathbf{R}^{d}$. Furthermore, if we define $\mathscr{T} \equiv\{F: F$ in $\mathscr{F}$ and $D \subseteq F\}$, then $D=\cap\{F: F$ in $\mathscr{H}\}$. Members of $\mathscr{K}$ may be indexed $H_{1}, H_{2}, \ldots$ For convenience of notation, we will let $J_{i}$ denote the supporting hyperplane for $\boldsymbol{H}_{i}$, $1<i<\infty$.

To begin the induction, select the first hyperplane $J_{1}$ and choose a point $x_{1}$ in $J_{1} \sim D \neq \varnothing$. Define $S_{1}=\operatorname{conv}\left(x_{1} \cup D\right)$. Choose a real number $f_{1}$ so that $0<f_{1}<\frac{1}{2} \operatorname{dist}\left(x_{1}, D\right)$, and let $T_{1}=\operatorname{conv}\left(x_{1} \cup N\left(D, f_{1}\right)\right)$, where $N\left(D, f_{1}\right)=\{z$ : $\left.\operatorname{dist}(z, D)<f_{1}\right\}$.

Inductively, assume that for $k \geqslant 2, x_{1}, \ldots, x_{k}, S_{1}, \ldots, S_{k}, f_{1}, \ldots, f_{k}$ and $T_{1}, \ldots, T_{k}$ are defined, where

$$
\begin{gathered}
S_{k}=\bigcup_{1<i<k}\left\{\operatorname{conv}\left(x_{i} \cup D\right)\right\}, \\
0<f_{k} \leqslant \frac{1}{2} \min \left\{\operatorname{dist}\left(x_{i}, \operatorname{conv}\left(x_{j} \cup D\right)\right): i \neq j, 1<i, j<k\right\}, \\
T_{k}=\bigcup_{1<i<k}\left\{\operatorname{conv}\left(x_{i} \cup N\left(D, f_{k}\right)\right)\right\},
\end{gathered}
$$

and

$$
x_{i} \notin \operatorname{conv}\left(x_{j} \cup N\left(D, f_{k}\right)\right) \quad \text { for } i \neq j, 1<i, j<k .
$$

We would like for hyperplane $J_{k+1}$ to meet int $N\left(D, f_{k}\right)$. If this does not occur, then by our choice of $\mathcal{H}$, there must be a halfspace $H_{n}$ in $\mathcal{K}, n>k+1$, for which the corresponding hyperplane $J_{n}$ is parallel to $J_{k+1}$ and for which $J_{n} \cap$ int $N\left(D, f_{k}\right) \neq$ $\varnothing$. Note that $H_{n} \subseteq H_{k+1}$. Remove $J_{k+1}$ and $H_{k+1}$ from our list and replace them with $J_{n}$ and $H_{n}$, respectively. Then renumber appropriate $J$ and $H$ sets so that their indices are consecutive. (That is, $J_{n}$ will be renamed $J_{k+1}$ and $J_{n+m}$ will be renamed $J_{n+m-1}$ for $m \geqslant 1$. Corresponding $H$ sets will be renamed, too.)

Now $J_{k+1} \cap$ int $N\left(D, f_{k}\right) \neq \varnothing$. Since $d>3$, we may prove that $J_{k+1} \cap T_{k} \sim S_{k}$ $\neq \varnothing$, and select point $x_{k+1} \in J_{k+1} \cap T_{k} \sim S_{k}$. Clearly $x_{k+1} \notin \operatorname{conv}\left(x_{i} \cup D\right)$ for $1<i<k$. Show that $x_{i} \notin \operatorname{conv}\left(x_{k+1} \cup D\right)$ for $1<i<k$, to conclude that $x_{i} \notin$ $\operatorname{conv}\left(x_{j} \cup D\right)$ for $i \neq j, 1 \leqslant i, j \leqslant k+1$.

Define $S_{k+1}=\cup_{1<i<k+1}\left\{\operatorname{conv}\left(x_{i} \cup D\right)\right\}$. Choose real number $f_{k+1}$ so that $0<f_{k+1}<\frac{1}{2} \min \left\{\operatorname{dist}\left(x_{i}, \operatorname{conv}\left(x_{j} \cup D\right)\right): i \neq j, 1<i, j<k+1\right\}$, and let $T_{k+1}=$ $\cup_{1<i<k+1}\left\{\operatorname{conv}\left(x_{i} \cup N\left(D, f_{k+1}\right)\right)\right\}$. Notice that by our choice of $f_{k+1}, x_{i} \notin$ $\operatorname{conv}\left(x_{j} \cup N\left(D, f_{k+1}\right)\right)$ for $i \neq j, 1 \leqslant i, j \leqslant k+1$. Observe that $T_{k+1} \subseteq T_{k}$ and that $\cup_{1<i<k+1} S_{i} \subseteq \cap_{1<i<k+1} T_{i}$. By induction, $S_{n}$ is defined for every $1<n$, and we let $S=\operatorname{cl}\left(\cup_{1<n} S_{n}\right)$. Using the facts that $\left\{f_{n}\right\}$ converges to zero and $x_{i} \notin$ $\operatorname{conv}\left(x_{j} \cup N\left(D, f_{n}\right)\right)$ for $1 \leqslant j \leqslant n, j \neq i$, one may show that each $x_{i}$ selected above sees via some $T_{n}$ (and hence via $S$ ) only points in the corresponding closed half space $H_{i}$. Then it is not hard to prove that set $S$ satisfies the theorem.

We close with an easy analogue of Theorem 3.

THeOREM 5. Let $D$ be any convex set in $\mathbf{R}^{d}$ which contains a hyperplane. Then $D$ is the kernel of some set $S \neq D$ in $\mathbf{R}^{d}$ if and only if there is a line $L$ in bdry $D$ with $L \cap D=\varnothing$. 
COROLlaRY 1. Let $D$ be a closed convex subset of $\mathbf{R}^{d}$ which contains a hyperplane. Then $D$ is not the kernel of any set $S \neq D$ in $\mathbf{R}^{d}$.

\section{REFERENCES}

1. Steven Lay, Proceedings of Conference on Convexity and Related Combinatorics, Dekker, New York, (to appear).

2. F. A. Valentine, Convex sets, McGraw-Hill, New York, 1964.

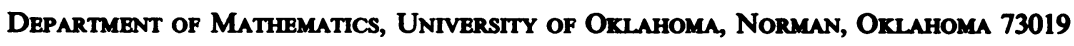

